\title{
Mean cell volumes of neutrophils and monocytes are promising markers of sepsis in elderly patients
}

\author{
A-Jin Lee, Sang-Gyung Kim \\ Department of Laboratory Medicine, Catholic University of Daegu, School of Medicine, Daegu, Korea
}

p-ISSN 2287-979X / e-ISSN 2288-0011 http://dx.doi.org/10.5045/br.2013.48.3.193 Blood Res 2013;48:193-7.

Received on March 26, 2013

Revised on May 31, 2013

Accepted on July 12, 2013

\author{
Correspondence to \\ Sang-Gyung Kim, M.D., Ph.D. \\ Department of Laboratory Medicine, \\ Catholic University of Daegu, School of \\ Medicine, 17-33, Duryugonwon-ro, \\ Nam-gu, Daegu 705-718, Korea \\ Tel: $+82-53-650-4145$ \\ Fax: +82-53-653-8672 \\ E-mail: sgkim@cu.ac.kr \\ (C) 2013 Korean Society of Hematology
}

\begin{abstract}
Background
Sepsis in elderly patients is a major cause of morbidity and mortality in the clinical setting. The aim of this study was to assess the diagnostic significance of volume conductivity scatter (VCS) parameters and to compare their reliability with that of inflammatory markers.
\end{abstract}

\section{Methods}

Patients $(\mathrm{N}=85)$ were divided into 3 groups according to their clinical history and culture results: control $(\mathrm{N}=29)$, localized infection $(\mathrm{N}=38)$, and sepsis $(\mathrm{N}=18)$. VCS parameters were obtained using a UniCel DxH 800 Coulter system. Cut-off values were established based on receiver operator characteristic (ROC) curves.

\section{Results}

The mean volumes of neutrophils (MNV) and monocytes (MMV) were higher in the sepsis group than in the localized infection and control groups ( $P=0.000$ for both). The mean cell conductivity and low median angle light scatter of neutrophils were lower in the sepsis group than in the localized infection and control groups $(P=0.029$ and $P=0.022$, respectively). With a cut-off of 156.5, MNV had a sensitivity of $83.3 \%$ and a specificity of $78 \%$ in predicting sepsis.

\section{Conclusion}

MNV and MMV, which can be obtained easily using an automated blood analyzer, may be promising hematologic parameters for distinguishing elderly individuals with and without sepsis and may help clinicians in the diagnosis of sepsis.

Key Words Volume of neutrophils, Volume of monocytes, VCS technology, Procalcitonin, Sepsis

\section{INTRODUCTION}

Sepsis in elderly patients remains a critical clinical issue because of the high rates of morbidity and mortality. More than $60 \%$ of sepsis patients are over 65 years of age [1]. The increasing incidence of sepsis among elderly individuals is a major public health concern. Therefore, early and accurate diagnosis is vital to ensure that appropriate medical decisions are made. To this end, clinicians in intensive care units and emergency departments need to predict systemic infection at an early stage. However, early diagnosis of sepsis is difficult because the signs and symptoms of sepsis in elderly patients, such as fever, are nonspecific and may be blunted or absent $[2,3]$. Therefore, laboratory findings are important in the diagnosis of sepsis.

Laboratory parameters such as WBC count, differential count, C-reactive protein (CRP) level, procalcitonin level, and blood culture results can provide valuable information for the diagnosis of sepsis. WBC count is routinely recommended as an initial screening marker. Elevated WBC and absolute neutrophil count as well as left-shifted neutrophils are associated with sepsis [4]. These parameters can be determined using an automated hematology analyzer, but the morphology of neutrophils should also be reviewed through peripheral blood (PB) smears. Immature granulocytes, such as band forms and reactive monocytes, tend to be larger and have lower nuclear complexity than their normal mature counterparts. PB smears, however, require manual examination and experienced medical technologists, and the results 
of PB smears are subjective because they depend not on instrument interpretation but on human interpretation. CRP has been shown to be a more sensitive and specific biomarker than WBC count [5]. Furthermore, procalcitonin levels distinguish bacterial from viral infections, correlate well with clinical severity $[6,7]$, and show higher predictive sensitivity than CRP [8]. Blood culture remains the gold standard for diagnosing septicemia; however, this method shows low sensitivity [9], requires an adequate amount of blood and proper collection techniques to avoid contamination, and can delay diagnosis as the results may require several days.

The latest automatic hematology analyzers, such as the Coulter DxH 800 (Beckman Coulter, Fullerton, CA), can use a variety of volume conductivity scatter (VCS) technologies. The VCS technology of the Coulter cell analysis system can directly obtain data from more than 8,000 WBCs using direct current impedance to measure cell volume (V) for accurate size of all cell types, radio frequency opacity to characterize conductivity (C) for internal composition of each cell, and a laser beam to measure light scatter (S) for cytoplasmic granularity and nuclear structure [10, 11]. In addition, a flow cytometry channel of the UniCel DxH 800 was introduced to provide better resolution and achieve new levels of performance [12]. This system can analyze individual cells using multiple angles of light scatter. There are 3 additional measurements of the scatter compared to that in previous Coulter models such as the LH750. Median angle light scatter (MALS), lower median angle light scatter (LMALS), and upper median angle light scatter (UMALS) provide information concerning granularity and the membrane surface, axial light loss measurement of cellular transparency, and the low angle light scatter (LALS) cellular complexity index, respectively [12]. An automated differential count using these data identifies each cell as a neutrophil, lymphocyte, monocyte, eosinophil, or basophil.

This study aimed at assessing the diagnostic significance of VCS parameters for sepsis in elderly patients and at comparing their reliability with that of inflammatory markers such as procalcitonin, CRP, and WBC count.

\section{MATERIALS AND METHODS}

\section{Patients}

We retrospectively analyzed data from 85 patients who were more than 50 years old. The 85 patients were divided into 3 groups according to their clinical history and culture results: patients without signs of infection $(\mathrm{N}=29)$, patients with localized infection ( $\mathrm{N}=38)$, and patients with systematic infection $(\mathrm{N}=18)$. Patients with systemic infection fulfilled the criteria of sepsis according to the American College of Chest Physicians (ACCP) guidelines [13]. Patients with localized infection presented with organ-specific infections such as pneumonia, urinary tract infection, acute pyelonephritis, and bronchopulmonary infections. Clinical signs (temperature $>38.5^{\circ} \mathrm{C}$ and purulent sputum) and positive results on chest radiography were the basis for diagnoses of pneumo- nia and bronchopulmonary infections. Clinical signs and positive results for urinary culture of significant pathogens defined urinary tract infection and acute pyelonephritis. Control patients $(\mathrm{N}=29)$ showed no symptoms of infection, such as fever, and had WBC and differential counts within normal limits. From all patients, blood samples for WBC count, differential count, and serum markers of inflammation levels were collected on the same day of blood culture collection. All septic patients $(\mathrm{N}=18)$ had positive blood cultures. Patients with systematic infection in which blood cultures yielded bacteria likely to be contaminants, such as coagulase-negative staphylococci, were excluded from the study.

\section{Complete blood count and cell population data assay}

Complete blood count (CBC) and cell population data (CPD) included WBC counts with automated differential counts and VCS parameters of neutrophils and monocytes. VCS parameters were obtained during the passage of each cell through the aperture and measured by the Coulter $\mathrm{DxH}$ 800 cellular analysis system. Such parameters reflected the mean neutrophil volume (MNV), mean neutrophil conductivity (MNC), and mean neutrophil scatter (MNS). MNS in turn consisted of MALS, UMALS, LMALS, and LALS. These parameters were also applied for the analysis of monocytes.

\section{C-reactive protein assay}

The level of CRP was obtained by a model 7600-110 automated chemistry analyzer (Hitachi, Tokyo, Japan) using an established immunoturbidimetric method. CRP values $\leq 5$ $\mathrm{mg} / \mathrm{L}$ were considered normal.

\section{Procalcitonin assay}

The level of serum procalcitonin was determined using an enzyme-linked fluorescent assay $\left(\right.$ VIDAS $^{\circledR}$ B.R.A.H.M.S PCT; bioMérieux, Marcy L'Etoile, France). The assay uses alkaline phosphatase-labeled mouse monoclonal immunoglobulins against human procalcitonin. The serum samples were mixed with conjugates, and emitted signals were detected and analyzed automatically. Procalcitonin levels $\leq 0.05 \mathrm{ng} / \mathrm{mL}$ were considered normal.

\section{Statistical analysis}

Statistical analysis was performed using the SPSS software, version 13.0 (SPSS, Chicago, IL, USA). Results are expressed as mean \pm standard deviation. Sepsis parameters were compared between the different groups using analysis of variance (ANOVA) and subsequent post hoc range tests. $P$ value $<0.05$ was considered statistically significant. The diagnostic properties of each test were investigated by receiver operator characteristic (ROC) curves. The optimum cutoff value for each variable was tested. The sensitivity, specificity, and area under the ROC curve were then assessed. 


\section{RESULTS}

\section{Clinical data}

The mean age was 67.6 years in patients with sepsis, 64.6 years in patients with localized infection, and 68.2 years in patients without symptoms of infection. Data on age and sex distribution are shown in Table 1. In the sepsis patients, the most commonly isolated pathogens were Escherichia coli and Staphylococcus aureus. WBC count ranged from $0.7 \times 10^{9} / \mathrm{L}$ to $25.9 \times 10^{9} / \mathrm{L}$, with a mean of $11.0 \times 10^{9} / \mathrm{L}$. The percentage of neutrophils ranged from $57.2 \%$ (0.572) to $97.4 \%$ (0.974), with a mean of $82.8 \%(0.828)$. All sepsis patients had clinical signs and symptoms of acute infection. The mean WBC count and percentage of neutrophils were $9.8 \times 10^{9} / \mathrm{L}$ and $74.7 \%$, respectively, in patients with localized infection and $8.1 \times 10^{9} / \mathrm{L}$ and $67.0 \%$, respectively, in patients without symptoms of infection. The highest mean CRP level was $151.0 \mathrm{mg} / \mathrm{L}$, which was found in the sepsis group and significantly differed from the mean values of the other 2 groups $(P=0.004)$. The procalcitonin levels were also the highest in the sepsis group $(53.03 \mathrm{ng} / \mathrm{mL})$ and were significantly different from those of the localized infection and patients without infection groups $(P=0.000$ and $P=0.000$, respectively).

\section{VCS parameters}

\section{Neutrophils}

MNV was the highest in the sepsis group (range, 138203) compared to that in the localized infections (range, $135-190)$ and control (range, $130-169)$ groups $(P=0.003$ and $P<0.001$, respectively). MNC was lower in the sepsis group (range, 135-154) than in patients without infection (range, 143-155; $P=0.048$ ), but no differences were noted between the values in the sepsis group and the localized infection group (range, 137-157; $P=0.847$ ). With regard to neutrophil scatter, low median angle light scatter of neutrophils was lower in the sepsis group (range, 116-145) than in patients without infections (range, 120-146), indicating an increase in immature granulocytes during sepsis $(P=0.015)$. However, this parameter did not significantly differ between the sepsis group and the localized infection group (range, 11-148; $P=0.123)$.

\section{Monocytes}

Mean monocyte volume (MMV) was the highest in the sepsis group (range, 172-217) compared to that in the localized infection (range, 158-208) and control (range, 158$210)$ groups $(P<0.001$ and $P<0.001$, respectively). Mean monocyte conductivity (MMC) and mean monocyte scatter

Table 1. Characteristics, parameters of infection and inflammation, and VCS parameters.

\begin{tabular}{|c|c|c|c|c|c|c|c|}
\hline & \multirow{2}{*}{$\begin{array}{c}\text { Group } 1 \\
\text { Sepsis } \\
(N=18)\end{array}$} & \multirow{2}{*}{$\begin{array}{c}\text { Group } 2 \\
\text { Localized infection } \\
(\mathrm{N}=38)\end{array}$} & \multirow{2}{*}{$\begin{array}{l}\text { Group } 3 \\
\text { Controls } \\
(\mathrm{N}=29)\end{array}$} & \multicolumn{4}{|c|}{$P^{\mathrm{a})}$} \\
\hline & & & & All groups & 1 vs. 2 & 1 vs. 3 & 1 vs. 3 \\
\hline Age (year) & $67.6 \pm 15.0$ & $64.6 \pm 15.1$ & $68.2 \pm 16.2$ & & & & \\
\hline Gender $(\mathrm{f} / \mathrm{m})$ & $6 / 12$ & $16 / 22$ & $17 / 12$ & & & & \\
\hline Procalcitonin (ng/mL) & $53.05 \pm 72.88$ & $2.95 \pm 6.60$ & $0.59 \pm 1.18$ & $<0.001$ & $<0.001$ & $<0.001$ & $<0.001$ \\
\hline CRP (mg/L) & $151.0 \pm 6.8$ & $98.0 \pm 74.9$ & $74.5 \pm 82.6$ & 0.004 & 0.047 & 0.003 & 0.05 \\
\hline $\mathrm{WBC}\left(\times 10^{3} / \mu \mathrm{L}\right)$ & $11.0 \pm 6.8$ & $9.8 \pm 4.5$ & $8.1 \pm 2.0$ & & & & \\
\hline Neutrophil (\%) & $82.8 \pm 13.5$ & $74.7 \pm 14.0$ & $67.0 \pm 7.2$ & & & & \\
\hline \multicolumn{8}{|l|}{ VCS parameters } \\
\hline \multicolumn{8}{|l|}{ Neutrophils } \\
\hline Volume & $165 \pm 24.4$ & $153 \pm 20.2$ & $148 \pm 19.3$ & $<0.001$ & 0.003 & $<0.001$ & $<0.001$ \\
\hline Conductivity & $146 \pm 6.4$ & $148 \pm 5.1$ & $150 \pm 5.3$ & 0.027 & 0.847 & 0.048 & 0.03 \\
\hline \multicolumn{8}{|l|}{ Scatter } \\
\hline MALS & $130 \pm 13.2$ & $132 \pm 13.3$ & $134 \pm 13.6$ & 0.294 & 0.700 & 0.271 & 0.607 \\
\hline UMALS & $138 \pm 13.1$ & $134 \pm 12.9$ & $134 \pm 13.2$ & 0.174 & 0.159 & 0.304 & 0.937 \\
\hline LMALS & $118 \pm 17.5$ & $125 \pm 17.4$ & $129 \pm 17.6$ & 0.020 & 0.123 & 0.015 & 0.473 \\
\hline LALS & $157 \pm 40.2$ & $174 \pm 39.0$ & $177 \pm 39.5$ & 0.101 & 0.264 & 0.084 & 0.699 \\
\hline \multicolumn{8}{|l|}{ Monocytes } \\
\hline Volume & $193 \pm 29.4$ & $177 \pm 23.0$ & $174 \pm 22.0$ & $<0.001$ & $<0.001$ & $<0.001$ & $<0.001$ \\
\hline Conductivity & $128 \pm 7.6$ & $128 \pm 6.1$ & $128 \pm 6.9$ & 0.603 & 0.750 & 0.997 & 0.627 \\
\hline \multicolumn{8}{|l|}{ Scatter } \\
\hline MALS & $81 \pm 13.4$ & $81 \pm 13.3$ & $82 \pm 13.4$ & 0.697 & 0.996 & 0.826 & 0.692 \\
\hline UMALS & $89 \pm 14.3$ & $89 \pm 14.2$ & $90 \pm 14.0$ & 0.837 & 0.830 & 0.959 & 0.938 \\
\hline LMALS & $68 \pm 16.8$ & $69 \pm 17.1$ & $70 \pm 17.5$ & 0.607 & 0.824 & 0.580 & 0.858 \\
\hline LALS & $101 \pm 20.0$ & $107 \pm 16.2$ & $106 \pm 16.7$ & 0.553 & 0.522 & 0.742 & 0.928 \\
\hline
\end{tabular}

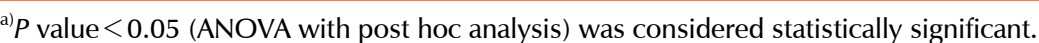

Abbreviations: CRP, C-reactive protein; VCS, volume conductivity scatter; MALS, median angle light scatter; UMALS, upper median angle light scatter; LMALS, lower median angle light scatter; LALS, low angle light scatter. 


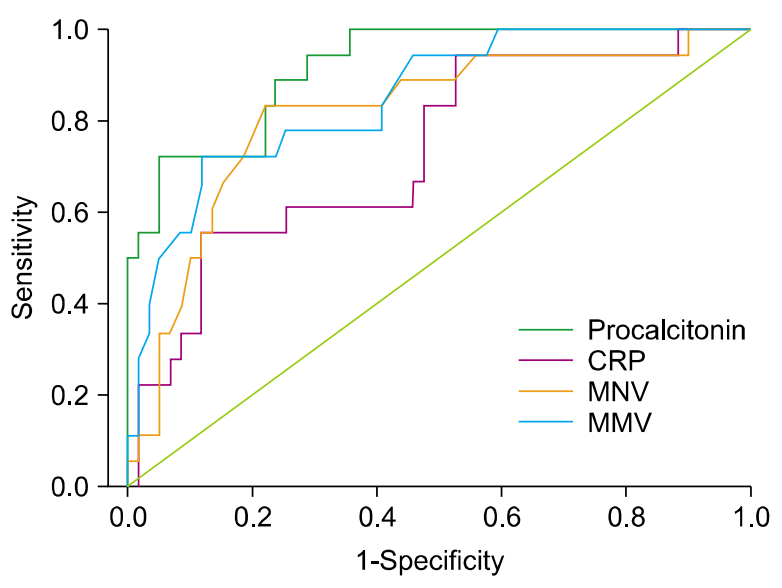

Fig. 1. Receiver Operator Characteristic (ROC) curves (with area under the ROC curve) of inflammatory markers (mean neutrophil volume $[\mathrm{MNV}]$, mean monocyte volume [MMV], and C-reactive protein [CRP]) and procalcitonin.

(MMS) did not differ between the 3 groups.

\section{Evaluation of sensitivity and specificity in predicting sepsis}

The sensitivity and specificity for inflammatory markers such as MNV, MMV, and procalcitonin were calculated at selected cut-off points. These data are shown in the ROC curves (Fig. 1) and Table 2.

When we selected a cut-off value for MNV that was $\geq 146.5$, we achieved a sensitivity of $94.4 \%$ and a specificity of $44.1 \%$. Although the sensitivity decreased to $83.3 \%$ using 156.5 as the MNV cut-off, the specificity improved to $78 \%$. Using 181.5 as the MMV cut-off, a sensitivity of $77.8 \%$ and a specificity of $74.6 \%$ were achieved. When we selected a cut-off value for MMV of $\geq 175.5$, the specificity decreased to $59.3 \%$ and the sensitivity increased to $83.3 \%$. Using 69.95 as the CRP cut-off, the sensitivity was $83.3 \%$ and the specificity was $52.5 \%$. Thus, at a cut-off of 156.5 for MNV and 175.5 for MMV, both parameters had equal sensitivity to that of CRP with a better specificity for predicting sepsis. In addition, MNV was a better predictive marker of acute bacterial infection than MMV. However, procalcitonin at a cut-off value of $0.32 \mathrm{ng} / \mathrm{mL}$ had a sensitivity of $94.4 \%$ with a specificity of $72 \%$ and appears to be the best predictive discriminator of all parameters assessed in the present study.

\section{DISCUSSION}

WBC count as well as the percentage of neutrophils and immature granulocytes can be measured using automated hematology analyzers and have been used to predict infection [4]. Manual PB examination can also provide useful diagnostic information such as morphologic changes in neutrophils, including toxic granulation, toxic vacuolization, and the presence of Döhle bodies [14]. However, manual slide differential counting is labor-intensive and time-consuming.
Table 2. Sensitivity and specificity at the designated cut-off values of inflammatory markers for predicting sepsis.

\begin{tabular}{lccc}
\multicolumn{1}{c}{ Parameter } & Cut-off values & Sensitivity (\%) & Specificity (\%) \\
\hline MNV & 156.5 & 83.3 & 78 \\
& 150.5 & 88.9 & 52.5 \\
MMV & 146.5 & 94.4 & 44.1 \\
& 181.5 & 77.8 & 74.6 \\
CRP (mg/L) & 175.5 & 83.3 & 59.3 \\
Procalcitonin (ng/mL) & 69.95 & 83.3 & 52.5 \\
\hline
\end{tabular}

Abbreviations: MNV, mean cell volume of neutrophils; MMV, mean cell volume of monocytes; CRP, C-reactive protein.

Furthermore, the 100-cell differential count has problems associated with imprecision, insensitivity, interobserver variation, and variation owing to the heterogeneous distribution of leukocytes on blood films [4].

Using automated hematology analyzers, we can obtain automated, accurate, and precise differential counts. These instruments allow high-throughput performance and rapid examination of very large numbers of cells to provide comprehensive hematology profiles. The typically obtained parameters are WBC with differential count, red blood cell counts, and hemoglobin, hematocrit, and reticulocyte levels. In addition, these tools can provide measures of variability for cells, such as red blood cell distribution width, cell size, and mean corpuscular volume [15]. VCS technology using newer automatic hematology analyzers has made it possible to analyze many WBCs automatically, which reduces the time for differential analysis and classifies cells with high efficiency [12].

In the present study, we demonstrated that morphologic changes in neutrophils (MNV) and monocytes (MMV) are observed in left-shifted reactive neutrophils and monocytes during sepsis in elderly patients and could be quantitatively evaluated by VCS technology using the UniCel DxH 800 hematology analyzer. We demonstrated that sepsis patients had higher MNV values and lower MNC and LMALS of MNS values than non-sepsis patients. However, only the MNV value could discriminate between the sepsis, localized infection, and control groups. We also showed that MMV was higher in the sepsis group than in non-sepsis patients. Therefore, MNV and MMV may be useful as discriminators to distinguish between sepsis and non-sepsis patients.

The use of VCS parameters in the clinic setting can provide several advantages. First, these parameters are determined during differential analysis without additional specimen requirements [14]. Second, determining these values is not labor-intensive or time-consuming, which are issues in manual differentiation. Finally, these values are more accurate, objective, and quantitative than manual differential counts because more than $8,000 \mathrm{WBCs}$ are evaluated automatically with the VCS instrument [11].

In this study, the clinical usefulness of VCS parameters was determined by evaluating the sensitivity and specificity 
of these parameters against CRP and procalcitonin, which are commonly used laboratory values for diagnosing bacterial infection and bacteremia. Of these indicators, procalcitonin had the highest sensitivity for predicting infection. However, MNV had the highest specificity and only a slightly lower sensitivity than procalcitonin. Measurement of CBC is typically routinely performed as part of the workup for sepsis patients. Therefore, MNV has the potential to be used as an additional marker of sepsis at a lower cost than the current markers.

The UniCel DxH 800 Coulter cellular analysis system contributes to improving differential accuracy and flagging efficiencies by combining additional light scatter measurements such as UMALS, LMALS, LALS, and AL. It reduces the working time and costs compared to previous automated hematology analyzers such as the Coulter LH 750 and 755 [12].

This study is a retrospective study and is limited by its small sample size and highly selected group. These limitations may have led to incorporation bias overestimating the diagnostic power of the investigated markers. Larger prospective cohort studies are needed to further validate the clinical usefulness of VCS parameters. Clinical scores with regard to organ failure and outcome such as the Acute Physiology and Chronic Health (APACHE) score should also be used to quantitatively evaluate the patient group. Other clinical applications of the VCS parameters should be evaluated in other neutrophilic leukocytosis, including pathological left shifts in cases of chronic inflammation, tumors, myeloproliferative neoplasms, and granulocyte colony stimulating factor stimulation. Further, whether the quality and accuracy of VCS data depend on certain techniques of a specific brand of automated hematology analyzers should be investigated.

In conclusion, MNV and MMV values, which are easily obtained by an automated blood analyzer, may be promising hematologic parameters for differentiating between sepsis and non-sepsis cases in elderly patients, as well as for helping clinicians diagnose sepsis.

\section{Authors' Disclosures of Potential Conflicts of Interest}

No potential conflicts of interest relevant to this article were reported.

\section{REFERENCES}

1. Angus DC, Linde-Zwirble WT, Lidicker J, Clermont G, Carcillo J, Pinsky MR. Epidemiology of severe sepsis in the United States: analysis of incidence, outcome, and associated costs of care. Crit Care Med 2001;29:1303-10.
2. Gleckman R, Hibert D. Afebrile bacteremia. A phenomenon in geriatric patients. JAMA 1982;248:1478-81.

3. Castle SC, Norman DC, Yeh M, Miller D, Yoshikawa TT. Fever response in elderly nursing home residents: are the older truly colder? J Am Geriatr Soc 1991;39:853-7.

4. Wile MJ, Homer LD, Gaehler S, Phillips S, Millan J. Manual differential cell counts help predict bacterial infection. A multivariate analysis. Am J Clin Pathol 2001;115:644-9.

5. Isaacman DJ, Burke BL. Utility of the serum C-reactive protein for detection of occult bacterial infection in children. Arch Pediatr Adolesc Med 2002;156:905-9.

6. Fernandez Lopez A, Luaces Cubells C, Garcia Garcia JJ, Fernandez Pou J. Procalcitonin in pediatric emergency departments for the early diagnosis of invasive bacterial infections in febrile infants: results of a multicenter study and utility of a rapid qualitative test for this marker. Pediatr Infect Dis J 2003;22:895-903.

7. Kourtis AP, Sullivan DT, Sathian U. Practice guidelines for the management of febrile infants less than 90 days of age at the ambulatory network of a large pediatric health care system in the United States: summary of new evidence. Clin Pediatr (Phila) 2004;43:11-6.

8. Yo $\mathrm{CH}$, Hsieh PS, Lee $\mathrm{SH}$, et al. Comparison of the test characteristics of procalcitonin to $\mathrm{C}$-reactive protein and leukocytosis for the detection of serious bacterial infections in children presenting with fever without source: a systematic review and meta-analysis. Ann Emerg Med 2012;60:591-600.

9. Procop GW, Hartman JS, Sedor F. Laboratory tests in evaluation of acute febrile illness in pediatric emergency room patients. Am J Clin Pathol 1997;107:114-21.

10. Krause JR. Automated differentials in the hematology laboratory. Am J Clin Pathol 1990;93(4 Suppl 1):S11-6.

11. Richardson-Jones A. An automated hematology instrument for comprehensive WBC, RBC, and platelet analysis. Am Clin Lab 1990;9:18-22.

12. Jean $A$, Boutet $C$, Lenormand $B$, et al. The new haematology analyzer $\mathrm{DxH}$ 800: an evaluation of the analytical performances and leucocyte flags, comparison with the LH 755. Int J Lab Hematol 2011;33:138-45.

13. Dellinger RP, Carlet JM, Masur H, et al. Surviving Sepsis Campaign guidelines for management of severe sepsis and septic shock. Crit Care Med 2004;32:858-73.

14. Chaves F, Tierno B, Xu D. Quantitative determination of neutrophil VCS parameters by the Coulter automated hematology analyzer: new and reliable indicators for acute bacterial infection. Am J Clin Pathol 2005;124:440-4.

15. Mardi D, Fwity B, Lobmann R, Ambrosch A. Mean cell volume of neutrophils and monocytes compared with C-reactive protein, interleukin- 6 and white blood cell count for prediction of sepsis and nonsystemic bacterial infections. Int $\mathrm{J}$ Lab Hematol 2010;32:410-8. 